ISSN: 0213-2079 - ISSN electrónico: 2386-3889

DOI: https://doi.org/10.14201/shhmo2020421287309

\title{
LA PRÁCTICA Y ENSEÑANZA DE LA ANATOMÍA EN LA REAL UNIVERSIDAD DE MÉXICO, SIGLOS XVI Y XVII
}

\section{Practice and Teaching of Anatomy in the Real Universidad de Mexico, 16th and 17th centuries}

\section{Gerardo MARTÍNEZ HERNÁNDEZ}

Instituto de Investigaciones Sobre la Universidad y la Educación (IISUE).

Universidad Nacional Autónoma de México

gemarh@yahoo.com

Fecha de recepción: 30/11/2019

Fecha de aceptación: 18/02/2020

RESUMEN: En este trabajo se estudian los inicios de la práctica anatómica en cuerpos humanos y su desarrollo durante los siglos XVI y XVII en la Nueva España. Antes de la instauración de la cátedra de anatomía en la Real Universidad de México, las disecciones anatómicas fueron muy comunes a lo largo de todo el virreinato. Durante la segunda mitad del siglo XVI médicos y cirujanos realizaban autopsias con diversos fines, lo que hace pensar que en la Nueva España llegó a haber una influencia de la reforma anatómica del Renacimiento. Posteriormente, en 1621 se institucionalizó la cátedra de anatomía en la universidad mexicana. Esto significó una mayor intervención sobre el desarrollo científico y la generación del conocimiento sobre el cuerpo humano y por lo tanto un control de los saberes anatómicos, que fueron acoplados al pensamiento contrarreformista.

Palabras clave: Medicina; anatomía; cirugía; Nueva España; siglos XVI y XVII.

ABSTRACT: This paper focuses on the first anatomical studies of human bodies and the development of the practice in New Spain during

Ediciones Universidad de Salamanca / అ@@ Stud. his., H. ${ }^{a}$ mod., 42, n. 1 (2020), pp. 287-309 
GERARDO MARTÍNEZ HERNÁNDEZ

LA PRÁCTICA Y ENSEÑANZA DE LA ANATOMÍA EN LA REAL UNIVERSIDAD DE MÉXICO, SIGLOS XVI Y XVII

the 16th and 17th centuries. Even before the Royal University of Mexico developed an anatomy chair, dissections were already very common throughout the Viceroyalty. During the second half of the 16th century, physicians and surgeons conducted autopsies for diverse purposes, suggesting that New Spain was influenced by the anatomical reform of the Renaissance. Later, in 1621, it was created the chair of anatomy and surgery in Mexican university, giving royal institutions greater control over scientific development and knowledge. As a result, anatomical studies stagnated, as a concession to Counter-Reformation doctrine.

Keywords: Medicine; anatomy; surgery; New Spain; $16^{\text {th }}$ and $17^{\text {th }}$ centuries.

\section{INTRODUCCIÓN}

El conocimiento del cuerpo humano y sus representaciones es un asunto que en las últimas décadas ha llamado la atención de distintos historiadores de la medicina y de la cultura (Cunningham, 1997; Carlino, 1999; Mandressi, 2012; Pardo, 2012; Skaarup, 2015). A través de los trabajos de estos investigadores se ha podido conjeturar que a pesar de su materialidad, el cuerpo es un objeto muy difícil de aprehender, puesto que sobre él se han elaborado discursos desde diversas temporalidades y esquemas de pensamiento. Cada cultura ha creado un razonamiento propio con el que ha reconocido su corporalidad y la relación que ésta ha tenido con su entorno y sus creencias. David Le Breton subraya que «Las representaciones de la persona y las del cuerpo, corolario de aquellas, están insertas en las visiones del mundo de las diferentes comunidades humanas» (Le Breton, 2002: 27). En el caso de la Nueva España, la hegemonía del pensamiento occidental logró consolidar una medicina académica mediante la imposición de su modelo cultural y la implantación de instituciones que dieron lugar a una práctica médica oficial. En este marco surgió la facultad de medicina de la Real Universidad de México a mediados del siglo XVI. La fase de consolidación académica e institucional de dicha facultad tuvo lugar entre los años 1553, año de apertura de la Real Universidad, y 1668, cuando entraron oficialmente en vigor los estatutos universitarios de Juan de Palafox y Mendoza. En este lapso de tiempo se dio un creciente interés por las disecciones anatómicas, que culminó con la apertura de la cátedra de anatomía y cirugía. Este trabajo se enfoca en la medicina galénica, en concreto en la práctica anatómica, que comenzó a ser de importancia fundamental para los médicos europeos a partir del siglo XVI. En la historiografía médica mexicana se ha prestado mucha atención a la anatomía durante el Renacimiento (Martínez, 2008; Pardo, 2012) pero no se ha dado seguimiento a su práctica durante la centuria siguiente. Por lo tanto, este trabajo pretende mostrar el proceso de institucionalización del saber anatómico en los círculos académicos

Ediciones Universidad de Salamanca / అ@@ Stud. his., H. ${ }^{a}$ mod., 42, n. 1 (2020), pp. 287-309 
de la Nueva España, que empezó con prácticas extrauniversitarias en la segunda mitad del XVI y culminó con la creación de una cátedra propia en el XVII. De este modo se pueden apreciar las particularidades del caso novohispano y su parcial desapego de los modelos europeos. En el periodo estudiado es posible percibir un acercamiento entre la ciencia y la técnica, que en el caso de la medicina enlazó a ésta con la cirugía, dando como resultado una nueva forma de conocer el cuerpo mediante la realización de disecciones humanas.

Para conocer el estado del conocimiento anatómico que se impuso en la Nueva España a partir del siglo XVI es preciso abordar la práctica de disecciones en la Edad Media y los cambios que el conocimiento anatómico sufrió a mediados de dicho siglo en algunos lugares de Europa, y que fueron asimilados en ciertos centros docentes de la Península Ibérica y posteriormente en el virreinato novohispano. De este modo se puede percibir que la anatomía practicada en la Nueva España estaba en estrecha relación con lo que sucedía en el ambiente europeo, sin embargo, no dejaba de tener las particularidades que el ambiente americano le daba.

Este estudio queda dividido en tres partes. En la primera se atiende el modo en que se practicaba la disección anatómica en la Nueva España durante el siglo XVI; en la segunda se abordan los antecedentes medievales y la reforma renacentista que transformó el conocimiento y la docencia de la anatomía en diversas universidades y que tuvo un influjo en la Nueva España; finalmente, en la tercera se muestran las evidencias y se explican los motivos por los cuales la anatomía novohispana del siglo XVII quedó apegada a la tradición escolástica.

\section{LA DISECCIÓN ANATÓMICA, UNA PRÁCTICA COMÚN EN LA NUEVA ESPAÑA DEL SIGLO XVI}

La enseñanza del conocimiento anatómico humano se institucionalizó en la Nueva España en el año de 1621 con la creación de la cátedra de cirugía y anatomía en la Real Universidad de México y años más tarde, en 1646, se consolidó con la primera demostración docente al interior de la facultad de medicina. Sin embargo, la práctica disectora ya tenía un tiempo considerable ejerciéndose con cierta regularidad en suelo novohispano. La primera referencia a la realización de una anatomía ${ }^{1}$, llevada a cabo en la ciudad de México, data de 1553, cuando el médico Cristóbal Méndez (c. 1500-c.1553) detalló en su obra Libro del ejercicio corporal (Sevilla, 1553) lo siguiente:

1. En este trabajo se referirá a la práctica de disecciones, de autopsias o de cualquier práctica de estudio anatómico post mortem con el término anatomía que comúnmente se utilizaba en la época.

Ediciones Universidad de Salamanca / 요 Stud. his., H. ${ }^{a}$ mod., 42, n. 1 (2020), pp. 287-309 
GERARDO MARTÍNEZ HERNÁNDEZ

LA PRÁCTICA Y ENSEÑANZA DE LA ANATOMÍA EN LA REAL UNIVERSIDAD DE MÉXICO, SIGLOS XVI Y XVII

Yo vide en México abrir a vn niño, hijo de vn muy honrrado hombre que se dezía Villaseñor, y no auía cinco años, y le sacaron una piedra casi tamaña como vn hueuo; y sin dubda, según su cantidad, que se hizo antes de que naciesse. ${ }^{2}$

Méndez estuvo en México entre los años de 1529 y 1545, tiempo en el que se puede situar la realización de la necropsia por él descrita. Igualmente, se sabe que en la década de 1540 el boticario Bartolomé de Nabira llevaba a cabo ciertas prácticas anatómicas en el Hospital Real de San José de Gracia en la ciudad de Querétaro (Pardo, 2012: 194).

Posteriormente, se encuentran varias noticias sobre las anatomías realizadas en el año de 1576 a raíz de la epidemia de cocoliztli, que afectó a gran parte del territorio novohispano. En este sentido destacan los informes que dejaron el Protomédico general Francisco Hernández (c.1514-1578) y el cirujano y mayordomo del Hospital de Indios Alonso López de Hinojosos (1534-1597). Este último refirió que

Sabido por el muy excelente señor virrey que los remedios de tan famosos médicos y sus pareceres no aprovechaban, mandó que se hiciesen anatomías; y por ser el hospital real más acomodado y donde hay mayor refrigerio que en toda la Nueva España por favorecerlo tan ampliamente como siempre lo favorece su excelencia por respeto de ser este bien para los naturales, y haber en el dicho hospital, en el dicho tiempo, más de doscientos enfermos de ordinario, y así se hicieron en él anatomías y yo propio por mis manos las hice estando presente el doctor Francisco Hernández, Protomédico de su majestad que al presente estaba haciendo experiencia de las yerbas medicinales, purgativas y otras cosas naturales de esta Nueva España, las cuales hacía por mandato de su majestad; el cual después de haber visto las anatomías que se hicieron dio noticia de ello a su excelencia. ${ }^{3}$

También hay una crónica fechada a finales del siglo XVI que señala al doctor Juan de la Fuente (c.1525-1595), primer catedrático de medicina en la Real Universidad de México, como uno de los médicos que «hizo anatomía de vn indio en el Hospital Real de México» (Dávila, 1625: 101) durante la epidemia de 1576. En la actualidad se ha puesto en duda la participación de Juan de la Fuente en las disecciones durante el cocoliztli, ${ }^{5}$ pues no existe ningún otro documento que compruebe la experiencia

2. (Méndez, 1996: 214-215)

3. (López de Hinojosos, 1977: 209).

4. La primera edición de esta obra de Dávila Padilla se hizo en Madrid en 1596.

5. Fue Germán Somolinos D’Ardois quien supuso falsa la imputación que se le ha hecho a Juan de la Fuente como participante en las autopsias de 1576. El argumento que esgrime Somolinos es que las anatomías que se realizaron en el Hospital de Naturales fueron promovidas por Francisco Hernández y llevadas a cabo por Alonso López de Hinojosos. Somolinos deduce que Dávila Padilla no conoció a fondo el trabajo de Hernández y que López de Hinojosos era un humilde cirujano, en cambio a finales del XVI, cuando Dávila Padilla escribía su crónica,

Ediciones Universidad de Salamanca / 요 Stud. his., H. ${ }^{a}$ mod., 42, n. 1 (2020), pp. 287-309 
anatómica de De la Fuente en aquel año. Sin embargo, basta revisar con atención los acontecimientos que se dieron en torno a dicha epidemia para suponer que efectivamente Juan de la Fuente pudo haber llevado a cabo algunas observaciones hechas de forma directa sobre las víctimas mortales del cocoliztli. De la Fuente fue médico del Hospital de Naturales, lugar en donde se realizaron autopsias. Además, se conoce que tenía en su biblioteca un ejemplar de la Fabrica de Vesalio y diversos textos de los autores más representativos del humanismo médico de la Universidad de Alcalá (Martínez, 2014: 207-211).

Pero la realización de anatomías durante el siglo XVI no se supeditaba solamente a casos emergentes como lo era la muerte de un miembro de una familia importante o las devastadoras epidemias. En los textos médicos de la época se puede leer que entre los médicos y cirujanos la disección de cadáveres era una práctica más común de lo que se podría pensar. En el texto de López de Hinojosos, cuya primera edición es de 1578, es frecuente hallar narraciones sobre las anatomías que él mismo realizaba en el Hospital Real de Naturales. En su libro hace hincapié en la utilidad que tiene el conocimiento de la anatomía humana basándose en la observación directa del cuerpo humano. «Esto lo he visto muchas veces en anatomías que he hecho por mis propias manos en el Hospital Real de Indios de esta ciudad» y «lo habemos visto en algunos que habemos hecho anatomías» (López de Hinojosos, 1977: 147 y 164) son algunas referencias que hace el cirujano en su obra.

Estas no son las únicas informaciones que ilustran la actividad anatómica que se desarrollaba en la Nueva España durante la segunda mitad del siglo XVI. En la segunda edición del Tratado breve de medicina (1592), su autor, fray Agustín Farfán (c. 1532-1604), expuso que había observado en el cráneo «la comisura en medio de este hueso. Y cuando se halla es en algunas mugeres; y yo lo he visto en casco de hombre» (Farfán, 1592: 327), lo que hace suponer que el médico agustino realizó prácticas anatómicas o fue testigo de algunas de ellas. A finales del siglo XVI Alonso López de Hinojosos brinda una singular estampa anatómica en la segunda edición de su Suma y recopilación de cirngía. En aquella ocasión apuntó que había hecho una anatomía

en una mujer que mataron preñada de tres o quatro meses vi todo esto que me admiré y estaba el niño sentado en el vientre, y el ombligo le dava una buelta al pescueço, que le servía de tenello derecho, y tenía la cara pegada con el ombligo de la madre. ${ }^{6}$

En esa misma edición el cirujano también refirió un caso que resulta de gran interés, pues muestra las circunstancias en que se dio aquella disección anatómica:

Juan de la Fuente era el prestigioso catedrático de medicina de la universidad. (Somolinos, 1980: 229-230).

6. (López de Hinojosos, 1595: 169r).

Ediciones Universidad de Salamanca / 요 Stud. his., H. ${ }^{a}$ mod., 42, n. 1 (2020), pp. 287-309 
En el año de mil y quinientos y noventa y dos, a siete de henero, murió un ciudadano de Guaxaca y, a contemplación de los demás ciudadanos y a pedimiento de los cirujanos, lo mandó abrir el alcalde mayor, que al presente era Luys Xuárez de Peralta, porque le dixeron que era un gran chupador de humo de piciete; y, con el deseo y bien común lo mandó abrir, y yo me hallé presente y vi que estaba su cuerpo como los que yo avía hecho anatomía diez y seis años antes, quando el gran cocolistle, porque tenía el hígado muy hinchado y los livianos secos y prietos, y el baço muy grande y duro, y las tripas prietas a trechos, y la hiel podrida y la diaflama y los demás miembros de dentro secos, sin ningún género de humidad, como si hubiera estado al humo por alguna distancia de tiempo. ${ }^{7}$

Resalta aquí la condición pública de esta anatomía, cuya finalidad era demostrar los daños nocivos de fumar tabaco en exceso. Seguramente a la disección descrita por López de Hinojosos asistieron distintos habitantes de la ciudad de Oaxaca, lo que sugiere que esta demostración anatómica no estaba destinada a un círculo de profesionales médicos, sino a un sector social más amplio. Esta autopsia trataba de demostrar lo perjudicial que era inhalar humo de tabaco de forma inmoderada. El protomédico Francisco Hernández, con quien López de Hinojosos tuvo una estrecha relación durante su estancia en la ciudad de México, ya advertía sobre las propiedades medicinales de esta yerba, pero también daba cuenta de que «debe evitarse [...] su uso inmoderado pues, de otra manera, se produce destemplanza cálida del hígado y sobreviene caquexia y otras enfermedades incurables» (Hernández, 1959: 80).

Todas las pruebas documentales presentadas sobre el ejercicio anatómico en la Nueva España del siglo XVI indican que no se trataba de prácticas docentes, es decir, no tenían fines didácticos en la formación de los médicos o cirujanos, ${ }^{8}$ pues la cátedra de cirugía y anatomía de la Real Universidad no fue abierta hasta 1621. Por lo tanto, las disecciones realizadas en suelo novohispano durante la centuria del XVI no pueden ser consideradas como prácticas supeditadas a la reforma anatómica renacentista iniciada por Andrés Vesalio (1514-1564), ya que se trataban de prácticas anatómicas de raigambre medieval a las que estaban muy habituados los médicos españoles. Aunque las anatomías que se realizaron en 1576 se han vinculado al movimiento vesaliano (Martínez, 2008), sus descripciones recuerdan más a la tradición escolástica en la que el médico, en este caso Francisco Hernández, dirigía al cirujano, Alonso López de Hinojosos, para que éste abriera y maniobrara los cuerpos. No obstante, existen indicios indiscutibles de que Francisco Hernán-

7. (López de Hinojosos, 1595: 150v-151r).

8. En este sentido se puede hacer referencia también a un caso que se dio en la Nueva Granada en 1608, en el que se mandó abrir el cuerpo de un tal Clemente Salguero para afirmar o negar si la causa de su muerte había sido por «yerbas», es decir, por un envenenamiento intencionado. En este caso la apertura del cuerpo fue llevada a cabo por un barbero para que los médicos pudieran observar el interior abdominal y emitir su juicio. (Ceballos, 1994: 227-231).

Ediciones Universidad de Salamanca / 요 Stud. his., H. ${ }^{a}$ mod., 42, n. 1 (2020), pp. 287-309 
GERARDO MARTÍNEZ HERNÁNDEZ

LA PRÁCTICA Y ENSEÑANZA DE LA ANATOMÍA EN LA REAL UNIVERSIDAD DE MÉXICO, SIGLOS XVI Y XVII

dez y muy probablemente Juan de la Fuente estuvieron influidos por la corriente anatómica vesaliana en algunos centros docentes de la Península.

\section{LA REFORMA ANATÓMICA: DEL SABER MEDIEVAL A LA MEDICINA HUMANISTA DEL RENACIMIENTO}

Algunos historiadores ya han señalado la diferencia que había en la Edad Moderna entre la anatomía con fines corroborativos y la que tenía objetivos didácticos. La primera se practicaba en Europa al menos desde el siglo XIII (Pardo, 2012: 189) y llegó a la Nueva España durante la primera mitad del siglo XVI; su finalidad era simplemente comprobar los diagnósticos predichos por los galenistas. Los médicos europeos que llegaron a tierras americanas inmediatamente después de la conquista tenían inquietudes de confirmar sus dictámenes y errores mediante la autopsia. Esta práctica continuó siendo común en la Nueva España durante los dos siglos siguientes. La segunda clase de anatomía venía gestándose a la par de la primera en los últimos siglos medievales y tuvo un florecimiento en el siglo XV y para la segunda mitad de la centuria posterior permitió un desarrollo sin parangón en los conocimientos anatómicos. El trabajo De bumanis corporis fabrica (1543) de Vesalio se convirtió, según algunos historiadores de la medicina, en el texto paradigmático de esta revolución anatómica renacentista 9 .

En el mundo medieval la teoría sobre la composición del cuerpo humano estaba basada en el tratado De usu partium, del médico griego Galeno de Pérgamo (130216), el cual prestaba mayor atención a la anatomía funcional, por lo que a la parte morfológica no se le daba gran importancia. Este tratado, cuyo título se podría traducir como Del uso de las partes, fue el primer estudio acerca de la anatomía y la única obra de referencia anatómica hasta el Renacimiento. ${ }^{10} \mathrm{El}$ estudio de este texto predominaba en las universidades, en donde la escolástica no admitía el ejercicio de la cirugía en la enseñanza de la medicina. Dentro del currículo universitario medieval

9. En este sentido vale la pena apuntar las revisiones que ha habido acerca de la figura de Vesalio y su aportación al conocimiento de la anatomía humana. En los últimos años se ha propuesto que la imagen histórica del médico flamenco ha sido idealizada, lo que ha conllevado a una mala interpretación de sus aportes reales al conocimiento anatómico. Es el caso de Juan José Barcia Goyanes, quien estipula que antes de Vesalio ya había autores que habían dado cuenta de los errores de Galeno y que varias de las láminas utilizadas en la Fabrica contienen errores anatómicos (Barcia, 1994).

10. La elaboración de este tratado se sitúa en torno a los años 164 y 175 cuando Galeno estuvo en Roma y en Aquilea, lugares en donde perfeccionó su conocimiento anatómico mediante la práctica de disecciones. El propósito de la obra, según el autor, es demostrar que cada parte del cuerpo tiene una función específica adecuada a la economía del cuerpo; señala que la efectividad de la estructura de cada una de las partes se manifiesta en la armonía de la acción de todo el órgano. (Galeno, 2010).

Ediciones Universidad de Salamanca / అ@@ Stud. his., H. ${ }^{a}$ mod., 42, n. 1 (2020), pp. 287-309 
de la medicina, la anatomía se redujo al estudio de los tratados galénicos, a la obra del cirujano medieval Guido de Chauliac (1300-1368), en disecciones animales y, eventualmente, en alguna humana. ${ }^{11}$

El detallado conocimiento de la anatomía humana no era considerado necesario por la medicina humoral debido a la metodología de su enseñanza. La escolástica era un método didáctico basado en el razonamiento argumentativo que intentaba eliminar las discrepancias entre los diversos autores mediante la demostración lógica. Este método sólo buscaba la reconciliación racional con el objetivo de crear un sistema único y coherente, en este caso el llamado sistema galénico. Por su propia naturaleza -de estar basado en textos e interpretaciones- el galenismo se alejaba de la observación directa de los fenómenos al interior del cuerpo humano. Cuando los argumentos de las autorictas se contradecían o la práctica anatómica presentaba alguna evidencia que refutara a las autoridades, inmediatamente se recurría a interpretar el problema de una manera adecuada y dentro de los límites teóricos (Grendler, 2002: 315-316).

La enseñanza de la anatomía en las universidades medievales se fundamentaba en los tratados galénicos y en las obras de algunos cirujanos y autores árabes. El catedrático universitario no realizaba por propia mano las disecciones. Esta tarea la ejecutaba un cirujano, siguiendo las instrucciones que el catedrático leía. Esta tradicional forma de estudiar el cuerpo humano comenzó a cambiar en el siglo XIV en algunos centros de enseñanza, entre los cuales destacaron las universidades de Montpellier, Bolonia y Padua.

Entre los siglos XIII y XIV en la Universidad de Montpellier, ligada a la corona de Aragón, se hicieron varias contribuciones al conocimiento médico. Anteriormente, entre los siglos X y XIII, la Escuela de Medicina de Salerno había gozado de gran prestigio. Parte de su reconocimiento se debió a la práctica de la cirugía. El decaimiento de la escuela salernitana coincidió con el auge de algunas universidades de la Península Itálica. Entre los siglos XIV y XV comenzaron a despuntar las universidades de Bolonia y Padua, lugares en los que se formaron varios comentaristas de Galeno, Avicena y Rhazes (865-925). Sin embargo, la aportación más valiosa de estos centros fue la autorización de la práctica de disecciones anatómicas en cadáveres humanos, las cuales tenían la intención de comprender las descripciones de Galeno. Aunque las universidades italianas fueron las primeras en incorporar las disecciones

11. Por ejemplo, todavía a mediados del siglo XVI, al fundarse la cátedra de anatomía en la Universidad de Salamanca se estipuló que el catedrático leyera a Galeno y Guido y estaba obligado a hacer treinta anatomías en cuerpos humanos o de animales cada año. Unos años después, en 1566, el claustro universitario salmantino ordenó que en la cátedra de cirugía se hicieran disecciones «en algún perro, o en algún cochino o en otro animal». (Santander, 1965: 203).

Ediciones Universidad de Salamanca / 요 Stud. his., H. ${ }^{a}$ mod., 42, n. 1 (2020), pp. 287-309 
GERARDO MARTÍNEZ HERNÁNDEZ

LA PRÁCTICA Y ENSEÑANZA DE LA ANATOMÍA EN LA REAL UNIVERSIDAD DE MÉXICO, SIGLOS XVI Y XVII

anatómicas humanas, su enseñanza todavía se supeditaba a los lineamientos dictados por el galenismo.

Durante la primera mitad del siglo XVI, en los círculos médicos europeos, comenzó a gestarse un marcado interés por el conocimiento de la anatomía del hombre basado en la observación. En este contexto apareció la figura de Andreas Vesalio. Vesalio estudió en París y Lovaina. En la época en que Vesalio estaba en París, hubo profesores de la facultad de medicina que realizaban anatomías sin carácter oficial en poblados cercanos a dicha ciudad (Barón, 1970: 19-25).

En 1537 Vesalio partió a Italia, primero a Venencia y luego a Padua, que era uno de los centros universitarios en donde se llevaban a cabo regularmente disecciones en cadáveres humanos. A finales de aquel año el bruselense fue nombrado catedrático de anatomía en Padua, hecho que le dio la oportunidad de ejercer regularmente demostraciones anatómicas. A diferencia de muchas otras universidades europeas, Padua contaba con un anfiteatro anatómico construido en 1525, además, su universidad era un centro en donde confluían alumnos de distintas procedencias, por lo que gozaba de un ambiente culturalmente rico y tolerante en el que era posible la convivencia y el intercambio de ideas. Las lecciones de Vesalio en Padua rompieron con la forma tradicional de enseñar la anatomía. El catedrático ya no dictaba la lección desde su estrado, dirigiendo las acciones del cirujano, sino que se situaba al lado del cadáver y él mismo hacía las disecciones para que los alumnos pudieran mirar la composición del cuerpo humano. El acto de acercarse al cadáver y alejarse de los libros hizo que la observación realizada por el catedrático y los alumnos cobrara una mayor relevancia en el aprendizaje.

En Venecia y en Padua, Vesalio pudo finalizar su obra cumbre: De humanis corporifabrica libri septem o Siete libros acerca de la estructura del cuerpo humano. ${ }^{12}$ Este tratado se imprimió por primera vez en junio de 1543 en Basilea. La obra de Vesalio ha trascendido en la historia por las descripciones del cuerpo humano basadas en la disección de cadáveres humanos. Vesalio era un fiel seguidor de Galeno, pero su trabajo comenzó a cuestionar las teorías anatómicas de éste. El Renacimiento fue una época de redescubrimiento y purificación filológica de los autores clásicos, pero también fue una etapa en la que el hombre comenzó a tener una mayor confianza en la observación de los fenómenos. De estas dos actividades resultó una nueva y a veces contradictoria interpretación del mundo natural, en este caso específico, de la constitución física humana.

La fama de los estudios médicos de la universidad patavina y el prestigio de Vesalio atrajeron a varios alumnos españoles, entre los destacaron el palentino Juan

12. Aunque se ha aducido que la palabra fabrica en el contexto del latín del Renacimiento tenía más una acepción de movimiento. Esto le daría una connotación más de carácter fisiológico que anatómico.

Ediciones Universidad de Salamanca / అ@@ Stud. his., H. ${ }^{a}$ mod., 42, n. 1 (2020), pp. 287-309 
Valverde de Amusco (1525-1588) y los valencianos Pedro Jimeno (1515-1551) y Luis Collado (1520-1580). Valverde, después de su experiencia en Padua, hizo su carrera como médico en distintas ciudades italianas y escribió el tratado anatómico Historia de la composición del cuerpo bumano (1556). En cambio, Jimeno y Collado retornaron a tierras ibéricas para poner en práctica lo aprendido en las lecciones anatómicas de Vesalio. Así, estos dos personajes se convirtieron en los introductores de la renovación anatómica en algunas universidades españolas. (López Piñero, 1976: 61-139). En 1547 la Universidad de Valencia contrató como catedrático de anatomía a Pedro Jimeno, quien estuvo al frente de la cátedra por tres años. En 1550 Jimeno se trasladó a la Universidad de Alcalá para hacerse cargo de la misma cátedra. La Universidad de Alcalá, fundada bajo el espíritu humanista, gozaba del ambiente propicio para que Jimeno desarrollara sus habilidades como docente de anatomía. En la facultad de medicina de Alcalá se formaron algunos de los más famosos cirujanos de la época como Francisco Arceo (1493-1580), Francisco Díaz (1530-1590) y Juan Fragoso (1530-1597).

Al quedar vacío el lugar que dejó Jimeno en la facultad de medicina de la Universidad de Valencia, las autoridades municipales designaron a Luis Collado como su sustituto. Entre los alumnos de Collado en las aulas valencianas estaba Cosme de Medina (m. 1591), quien en 1551 arribó a Salamanca para hacerse cargo de la nueva cátedra de anatomía. Cosme de Medina estuvo diez cursos al frente de la cátedra, pero se debe acotar que las autoridades de la Universidad de Salamanca no estaban de acuerdo con la imposición real de abrir nueva cátedra, por lo que, para darle la cátedra a Cosme de Medina, el claustro condicionó que su lectura se hiciera siguiendo a Galeno y Guido y la realización de treinta anatomías en cuerpos humanos o en animales. Así se produjo una de las tantas paradojas que abundan en la ciencia española del Renacimiento, pues habiendo un hombre con formación vesaliana se vio obligado a enseñar una anatomía de tipo medieval. Por ejemplo, hay constancia documental de que en 1561 las lecciones de anatomía no se estaban llevando a cabo de la forma adecuada (Carreras, 2005: 100) aunque ese mismo año, los estatutos elaborados por Diego de Covarrubias para la universidad salmantina ordenaban que en la cátedra de anatomía se hicieran disecciones y, en caso de que no se pudieran realizar, se explicara con «estampas y figuras de Besalio». El claustro salmantino, por lo tanto, no aceptó las innovaciones anatómicas que se estaban adoptando en otras universidades españolas.

Sin embargo, algunos discípulos de Cosme de Medina mostraron interés por el ejercicio anatómico. Tal fue el caso del catalán Francisco Micó (1528-1592), quien ejerció la medicina y herborizó en el Hospital de Guadalupe en Extremadura. El auge de la práctica médica en Guadalupe coincidió con el breve esplendor del movimiento anatómico en España. En un inventario de la biblioteca del monasterio, realizado en 1555, aparece anotada la Fabrica de Vesalio (López Piñero, 1976: 110).

Ediciones Universidad de Salamanca / అ@@ Stud. his., H. ${ }^{a}$ mod., 42, n. 1 (2020), pp. 287-309 
A mediados del siglo XVI, en Guadalupe se encontraba haciendo una estancia el médico Francisco Hernández, quien en 1570 fue investido por el rey Felipe II como Protomédico General de todas las Indias, Islas y Tierra Firme del Mar Océano y enviado al Nuevo Mundo para realizar la primera expedición científica en tierras americanas. En los comentarios que Hernández hizo a la Historia natural de Plinio apuntó que tuvo perfeccionamiento y formación en anatomía en el Hospital de Guadalupe al lado de Francisco Micó. Aparte del interés común por la anatomía, ambos también tenían curiosidad por el conocimiento de la aplicación de las plantas medicinales. Pero fue Hernández quien más destacó en esta disciplina al encomendársele la investigación y sistematización natural del Nuevo Mundo. Hernández, que además coincidió con Vesalio en la corte de Felipe II, llegó a tierras novohispanas a principios de 1571. El médico toledano traía consigo un amplio bagaje médico y cultural que puso a prueba durante su trabajo científico en la Nueva España, destacando la serie de anatomías que realizó en la Ciudad de México durante la epidemia de 1576.

El doctor Juan de la Fuente, primer catedrático de medicina de la Real Universidad de México, también tuvo un interés particular por la realización de estudios anatómicos. De la Fuente nació hacia 1525 en Sevilla. No se sabe con certeza dónde estudió, pero hay indicios que señalan que probablemente lo hizo en Alcalá o Salamanca (Martínez, 2014: 194). En una lista de los libros que De la Fuente llevó de España se encuentran los autores médicos más importantes de la universidad alcalaína, tales como Francisco Vallés, Fernando Mena (1520-1585) y Cristóbal de Vega, por lo que es probable que haya estudiado en esta universidad en la década de 1540. La única certeza que existe sobre el paso de De la Fuente por la universidad son sus grados de licenciado y doctor en Sevilla, los cuales le fueron otorgados en el verano de 1547 (Martínez, 2014: 179). En la carga de libros que llevó a Nueva España en 1564 se apuntó que De la Fuente llevaba un «Vesalio de Anathomía», además de otros libros sobre cirugía y anatomía (Martínez, 2014: 208).

Existe información que indica que Juan de la Fuente tuvo una educación acorde a los dictados humanistas de la época, no obstante, esto no es posible sostenerlo por la carencia de algún documento que lo corrobore, pues De la Fuente no legó ninguna obra escrita sobre medicina. Juan de la Fuente arribó a la Nueva España hacia 1562 con una formación humanista, pero al convertirse en catedrático de medicina de la Real Universidad en 1578 tuvo que sujetarse a los dictados de los primeros estatutos universitarios mexicanos, elaborados dentro de la ortodoxia tridentina traída a la Nueva España por Pedro Moya de Contreras, inquisidor y amigo personal de De la Fuente. Los estatutos de la Real Universidad de México, hechos en 1580, indicaban que en la única cátedra de medicina se tenía que leer «algo de Anatomia» (Jiménez, 1951: 45). Es probable que De la Fuente, como catedrático de medicina, haya intervenido en la redacción de esta disposición estatutaria. Acorde

Ediciones Universidad de Salamanca / అ@@ Stud. his., H. ${ }^{a}$ mod., 42, n. 1 (2020), pp. 287-309 
GERARDO MARTÍNEZ HERNÁNDEZ

LA PRÁCTICA Y ENSEÑANZA DE LA ANATOMÍA EN LA REAL UNIVERSIDAD DE MÉXICO, SIGLOS XVI Y XVII

a los estatutos de 1580 , los contenidos de la cátedra de medicina se ceñían más a un galenismo arabizado y la parte dedicada a la anatomía se basaba en el De usu partium, por lo que se puede deducir que la renovación anatómica renacentista no llegó, al menos de manera oficial, a la Real Universidad de México. No obstante, sí hay constancia de la presencia de médicos formados en el ambiente renovador del siglo XVI y que realizaron frecuentemente autopsias en diversos centros hospitalarios de la Nueva España. Habrá que esperar a la segunda década del siglo XVII para que en la Real Universidad se fundara la cátedra de cirugía y anatomía, sin embargo, para entonces, la situación de la medicina española y novohispana había cambiado de forma sustancial.

\section{EL ESTANCAMIENTO DE LOS SABERES ANATÓMICOS. LA INSTITUCIONALIZACIÓN DE LA ENSEÑANZA ANATÓMICA EN LA REAL UNIVERSIDAD DE MÉXICO}

En 1617, el rey Felipe III emitió una real pragmática, cuyo punto tercero establecía lo siguiente:

Que por quanto somos informados que de rresevir los estudiantes los grados de bachilleres, que es el ynportante, y con él se les da lisençia para curar por algunas universidades, donde no se lee ni ay cátredas de medicina, como son Yrache, Santo Tomás de Ávila, Osma y otras universidades semejantes, donde no se lee medeçina continuamente, y con ganar un curso en las unibersidades grandes llevando un testimonio los graduaban y açían bachilleres, y con eso se yban a curar sin tener çiençia ni experiencia. Mandamos que de aquí en adelante no se pueda dar grado de bachiller en ninguna unibersidad a ningún estudiante, sino fuere en las tres unibersidades principales o en las que por lo menos aya tres cátredas, de prima, de vísperas y la tercera de cirujía y anatomía, que entreanbas a dos cosas puede el catedrático de cirugía leer en sus tiempos. ${ }^{13}$

La redacción de este punto deja en claro los motivos por los que se implementó una serie de reformas a la educación médica en las universidades hispánicas al iniciar el siglo XVII. En la Época Moderna surgieron varias universidades en los territorios hispánicos. En 1475 había 8 universidades. Para 1625 existían 32. En dicha fecha, en los reinos de la corona de Castilla había 18, entre las que se contaban las universidades mayores de Salamanca, Valladolid y Alcalá. A la mayoría de las restantes se les calificaba de universidades «menores», las cuales se caracterizaban por un número reducido de estudiantes y el bajo costo de las matrículas y grados y, por ende, se dudaba de su nivel académico (Rodríguez-San Pedro, 2010: 33) Dicha

13. Archivo General de Indias (AGI), Escribanía, 168b, f. 7v.

Ediciones Universidad de Salamanca / అ@@ Stud. his., H. ${ }^{a}$ mod., 42, n. 1 (2020), pp. 287-309 
problemática, en el caso de la medicina, significaba un alto costo, pues lo que estaba en juego era la salud de los súbditos. La exigencia, por lo tanto, era clara: si alguna universidad perteneciente a los reinos hispánicos quería otorgar grados de bachiller en medicina tenía que tener al menos las cátedras de prima, vísperas y cirugía y anatomía en funciones. ${ }^{14}$

Al momento de la emisión de la pragmática, en la Real Universidad de México sólo existían las cátedras de prima (1578) y vísperas (1598). Dicho documento fue hecho público en la Universidad de México en el mes de marzo de 1621. Así, se hizo obligatoria la apertura de la cátedra de cirugía y anatomía. Sin embargo, la creación de dicha cátedra no iba a resultar tan sencilla por el hecho de que meses atrás se había abierto la cátedra de Metodo medendi, cuya lectura estaba a cargo de Cristóbal de Hidalgo Vendabal (c. 1580-1645).

A pesar del intento de la universidad por justificar la instauración de la cátedra de Metodo en lugar de la de cirugía y anatomía, la orden real era explícita: la tercera cátedra que se exigía debía de ser la de cirugía y anatomía. Así lo entendió el doctor Rodrigo Muñoz (?) y fue la razón que lo llevó a ofrecerse como lector de dicha cátedra. En octubre de ese año el doctor Muñoz puntualizó que «si en esta universidad no hubiese precisamente la dicha cátedra de cirugía y anatomía todos los grados que se diesen serían nulos de que resultarán muy grandes inconvenientes» (Fernández, 1953: 128).

El rector de la universidad, entonces, solicitó al doctor Muñoz que presentara pruebas y testigos de que tenía las aptitudes suficientes para que se le confiara la cátedra que él solicitaba. Ante esta petición, el interesado respondió que

Habiendo pedido que Vm. Se sirviese de nombrarme por catedrático de la cátedra de cirugía y anatomía, que precisamente se ha de erigir y criar en esta Real Universidad por estar así ordenado por nueva pragmática de su majestad, Vm. [...] cierto es que mi persona es más suficiente y idónea que no la dé un cirujano graduado, pues a los tales, en casos de peligro se les manda por reales pragmáticas y en sus títulos que se acompañen con médicos graduados; y pues yo hago servicio a esta Real Universidad, sirviéndola de gracia y todo viene a resultar en manifiesta utilidad de ella, y en cumplimiento de lo que está mandado por su majestad. ${ }^{15}$

La facultad de medicina no prestó atención a las exigencias del doctor Muñoz, ante lo cual el siguiente paso de este médico fue descalificar al catedrático Cristóbal

14. La expedición de la real pragmática de 1617, sin embargo, no significó su aplicación. Por ejemplo, la Universidad de Irache, una de las señaladas en la pragmática de llevar a cabo malas prácticas en su facultad de medicina, nunca fundó una sola cátedra en esta disciplina y siguió otorgando grados a médicos hasta la segunda mitad del siglo XVIII (Serrano: 2019).

15. (Fernández, 1953:129-130). 
Hidalgo, aduciendo que no había obtenido su cátedra mediante un proceso de oposición, y que la cátedra de Metodo se podía considerar simplemente como una lección extraordinaria y no obligatoria para los cursantes de medicina. Pero el fundamento con mayor peso que esgrimió fue que la lectura de Metodo no era lo que su majestad «tiene ordenado y mandado por su real pragmática» (Fernández, 1953: 132).

Ante la afrenta, el catedrático de Metodo decidió responder, asegurando que no era necesaria la fundación de una nueva cátedra, puesto que en su lección ya se enseñaba la cirugía y la anatomía. A dicha respuesta se sumó la orden del rector para que Hidalgo Vendaval

Lea en la dicha Real Universidad a la hora señalada, en lugar de la lectura de Methodo medendi, la cátedra de anatomía y cirugía, y de esta lectura se entienda el dicho auto del dicho doctor don Hernando Villegas, para que se cumpla de tener de la dicha real pragmática. ${ }^{16}$

Así, finalmente, se solucionó un conflicto que ya llevaba varios meses. El 29 de noviembre de 1621 se hizo oficial el nombramiento de Hidalgo Vendabal al frente de la cátedra de cirugía y anatomía.

Cinco años más tarde, el virrey marqués de Cerralvo (1580-1640) intentó imponer un corpus estatutario en la Real Universidad de México (González, 1991) cuestión que no prosperó, sin embargo, de esa tentativa legislativa emanó un documento que en algún momento se pensó sería el instrumento que regiría a la universidad. A través de este manuscrito se puede leer que para la cátedra de cirugía se había pensado que

Los primeros dos años leerá de los libros de Galeno De usu partium los que les pareciere son más menesterosos para saber el huso de cada partícula, pues en el principio de cada libro sumariamente lo ponga Galeno.

Los otros dos años leerá la sirujía meramente siguiendo a Guido, antiguo y docto barón, que se fundó con dotrina de Galeno y de Ippócrates y Avizena, y siga su modo en el leer por capítulos. ${ }^{17}$

De nueva cuenta, como en los anteriores estatutos, se recomendaba leer a las autoridades clásicas de la cirugía y de la anatomía: Galeno y Guido. No obstante, el contexto de la redacción de esta disposición es bastante diferente al ambiente que privaba medio siglo antes.

Como ya ha sido señalado, a partir del siglo XVI la cirugía comenzó a apoyarse en los conocimientos emanados del movimiento anatómico renacentista sin dejar de ser una profesión empírica. El cirujano estaba separado social y económicamente del médico universitario. Aunque esta situación era algo distinta en algunas universidades

16. (Fernández, 1953: 137).

17. (González, 1991: 85).

Ediciones Universidad de Salamanca / 요 Stud. his., H. ${ }^{a}$ mod., 42, n. 1 (2020), pp. 287-309 
de Italia y España. Por ejemplo, en la Península Ibérica hubo médicos consagrados a la práctica de la cirugía que desarrollaron su actividad en universidades, hospitales y ejército. La situación de la cirugía sufrió una notable transformación a partir de la segunda mitad del siglo XVI. Este cambio se debió a que los médicos con interés en la cirugía comenzaron a desplazar el saber bajomedieval de la enseñanza y práctica quirúrgicas. En sus tratados lograron integrar su experiencia profesional, dando una nueva sistematización a la teoría quirúrgica. En este contexto, los saberes académicos fueron una de las áreas en donde comenzó a darse un gradual ruptura con lo tradicional (López Piñero, 1979: 360).

La aproximación entre medicina y cirugía, o dicho de otra manera entre ciencia y técnica, que caracterizó a los saberes de la época moderna, fue un fenómeno que se trasplantó a los círculos académicos de los virreinatos americanos. Ya se ha abordado el caso del cirujano Alonso López de Hinojosos, quien escribió un arcaico texto de cirugía en la Nueva España de la segunda mitad del siglo XVI, sin embargo, su labor quirúrgica iba más allá de lo que se puede leer en su obra, ya que, por iniciativa propia o por petición de algunos médicos, solía hacer recurrentemente disecciones, como las realizadas a raíz de la epidemia de 1576.

La situación de la ciencia española de finales del siglo XVI y principios del XVII se ve reflejada en el caso particular de la enseñanza de la medicina en México. La facultad médica de la Real Universidad heredó los postulados de los avances científicos del siglo XVI e hizo obligatoria la cátedra de cirugía y anatomía, sin embargo, también fue presa de la paralización en que se había sumido la actividad científica española a partir de las disposiciones emanadas del pensamiento contrarreformista. Esto queda demostrado al ver que en el siglo XVII, en la cátedra de cirugía y anatomía, se retomaron como autoridades a Galeno, Guido y Avicena.

Esta contradictoria situación académica no hizo sino acrecentarse en los años siguientes. En la década de 1640, el emisario real, Juan de Palafox y Mendoza realizó una visita a la Real Universidad que tenía como finalidad reorganizar el estudio. Palafox tuvo un profundo interés por la formación de los médicos. Una revisión de los estatutos que resultaron de su visita a la universidad revela que el grado de bachiller en medicina fue meticulosamente regulado (Martínez, 2009). Entre las obligaciones que impuso Palafox a los estudiantes de medicina estuvo la de presenciar cada cuatro meses una anatomía que se haría en el Hospital Real de Naturales. En caso de no obedecer esta disposición, los catedráticos se harían acreedores a una sanción de 150 pesos y los estudiantes perderían los cursos hechos durante el año lectivo (Estatutos, 1668: 25).

Los estatutos palafoxianos fueron promulgados y publicados en 1668, sin embargo, las prácticas anatómicas con finalidad docente habían iniciado durante su visita a la universidad. En octubre de 1646, el cirujano del Santo Oficio y «ministro mayor de la anatomía» de la Real universidad, Juan de Correa (?)

Ediciones Universidad de Salamanca / 요 Stud. his., H. ${ }^{a}$ mod., 42, n. 1 (2020), pp. 287-309 
GERARDO MARTÍNEZ HERNÁNDEZ

LA PRÁCTICA Y ENSEÑANZA DE LA ANATOMÍA EN LA REAL UNIVERSIDAD DE MÉXICO, SIGLOS XVI Y XVII

Puso en execusión por sí sólo lo que xamás se había hecho que fue una anatomía en el Hospital de Nuestra Señora de esta ciudad, a los ocho de octubre de dicho año de seiscientos cuarenta y seis, donde asistieron los protomédicos, cirujanos, bachilleres y practicantes de ambas facultades con la aprobación de todos. ${ }^{18}$

Basta con revisar las circunstancias en las que se oficializó la docencia anatómica en la Real Universidad para advertir que las prácticas disectoras que comenzaron a llevarse a cabo a mediados del siglo XVII representaban una regresión a la doctrina escolástica, en la que el catedrático, en ese entonces Juan de Torres Moreno (c. 1623-1687), no hacía personalmente las disecciones, sino que éstas eran llevadas a cabo por el cirujano Juan de Correa. Se mantenía así la jerarquía que ponía a la medicina sobre la cirugía.

Correa fue un cirujano con intensa actividad en el virreinato novohispano, quien además de trabajar para la Inquisición, también laboró en las minas de Zacatecas y atendió a personajes importantes de la ciudad de México. Basado en su experiencia en las minas y en la lectura de algunos autores contemporáneos escribió el Tratado dela qualidad manifiesta, que el mercurio tiene. En la segunda parte de su obra describe el caso de un paciente que padeció una enfermedad renal que lo llevó a la muerte, tras lo cual Correa realizó una autopsia que quedó detallada en las páginas de su obra (Rodríguez-Sala, 1998: 723-725).

En 1667 ocurrió la temprana muerte del sucesor de Torres Moreno en la cátedra de cirugía y anatomía, el doctor Juan Germán Biros (1635-1667). Su cargo lo ocupó Diego de Osorio y Peralta (1635-1690), quien estuvo al frente de la lección durante cinco años. El nombre de este médico es importante para el desarrollo de la práctica anatómica en la Nueva España debido a que en 1685 publicó el primer tratado de anatomía con fines docentes para los estudiantes de la Real Universidad.

Diego de Osorio y Peralta fue un médico novohispano que realizó una larga carrera dentro de la facultad de medicina de la Real Universidad. Su trayectoria refleja el grado de consolidación que había adquirido la medicina académica en la Nueva España. Osorio y Peralta nació en la capital del virreinato en 1635 en el seno de una familia acomodada. Sus primeros estudios los realizó en el colegio de San Ildefonso. Luego, debió haber obtenido el grado de bachiller en artes por suficiencia entre 1650 y 1655 . En mayo de 1658 obtuvo el grado de bachiller en medicina. ${ }^{19}$ En 1662 se graduó como licenciado y doctor en medicina (Fernández de Recas, 1956: 67). Entre 1667 y 1688 regentó las cátedras de cirugía y anatomía, de Metodo y de vísperas $^{20} \mathrm{Al}$ mismo tiempo que desarrollaba su carrera como médico, Osorio y Peralta se convirtió en presbítero de la catedral metropolitana.

18. (Fernández, 1953:161-162).

19. Archivo General de la Nación (AGN), Ramo Universidad (RU), v. 279, ff. 374-377.

20. AGN, RU, v. 89 , ff. 434-439, 452-501 y $548-555$.

Ediciones Universidad de Salamanca / అ@@ Stud. his., H. ${ }^{a}$ mod., 42, n. 1 (2020), pp. 287-309 
Por su larga trayectoria, en 1685, Osorio y Peralta pasó a formar parte del Tribunal del Protomedicato como tercer Protomédico. ${ }^{21}$ Durante su gestión como autoridad sanitaria se vio involucrado en un caso inquisitorial, cuando dos boticarios, a quienes había hecho una visita como parte de sus funciones, lo acusaron de ejercer la medicina teniendo un cargo eclesiástico. Se sabe que en la época los eclesiásticos que ejercían la medicina debían de tener un permiso papal para ello y al parecer el tercer Protomédico no lo tenía. Durante el proceso salieron a la luz algunas diferencias que había entre los miembros del Protomedicato. ${ }^{22}$

La amplia experiencia acumulada a lo largo de varios años de servicio en la universidad y su preocupación por dar una óptima formación, según sus criterios, a los alumnos de la facultad de medicina impulsaron a Osorio y Peralta a escribir los Principia medicinae epitome et totius humani corporis fabrica. ${ }^{23} \mathrm{La}$ obra se encuentra escrita en latín y romance. La parte latina está dedicada a los cursantes de la facultad de medicina y la castellana a los cirujanos romancistas. En cuanto a su contenido, se puede decir que no cuenta con ninguna innovación, pues sigue al pie de la letra los cánones galenistas del neoescolasticismo, que se impuso en los territorios hispánicos como resultado de la Contrarreforma. Por ejemplo, basta leer la definición de anatomía que ofrece en las primeras páginas:

Anathomía es una recta división, o separación de los miembros simples, y compuestos del cuerpo humano, parte especulativa de la medicina, efecto provechosísimo de ella; sciencia adquirida por raciocinación demostrativa, por doctrina, y estudio de los libros, que es la theórica, y por la práctica, que da conocimiento a la manual operación por la experiencia de los cuerpos, que cortamos según Galeno, y todos los demás autores. ${ }^{24}$

21. AGI, México, 197, n. 42.

22. AGN, Inquisición, v. 667, f. 191.

23. Se trata de un libro en $4^{\circ}$ que consta de 104 fojas; está escrito en latín y castellano. Al inicio de la obra, el autor aclara que está dirigida a los cursantes de la facultad de medicina. Luego, viene el contenido del libro, el cual se encuentra dividido en siete partes: 1) Anatomia latino sermones (Anatomía en lengua latina), 2) Tractatus de partibus (Tratado sobre las partes), 3) Tractatus de diebus decretoris, de signis $\mathcal{E}$ de crisibus (Tratado de los días decretorios, de los signos y de las crisis), 4) De morbo Sancti Lazari Hispano idiomate (De la enfermedad de San Lázaro en idioma español), 5) Anathomia lingua nostra pro Chyrurgis non latinis (Anatomía en nuestra lengua para cirujanos no latinistas), 6) Septem Aphorismorum libri Hipocratis ex litera Leonisensi (Libro de los Siete Aforismos de Hipócrates según traducción de Leoniseno), 7) Tabula in qua omnes Aphorismi consiliatur cum morbis in particular pro insipientibus tam in teorica, quam in praxi necessaria (Tabla en la cual se consultan todos los aforismos con las enfermedades en particular. Para los principiantes tanto en la teoría como en la práctica). (Rodríguez-Sala, 1996: 71).

24. (Osorio y Peralta, 1685: ff. 60-61) Las cursivas son mías.

Ediciones Universidad de Salamanca / 요 Stud. his., H. ${ }^{a}$ mod., 42, n. 1 (2020), pp. 287-309 
En el siglo XVII novohispano no se podía hacer ningún tipo de interpretación teórica propia. El conocimiento tenía que estar reconocido y avalado por las autoridades políticas y religiosas. Cualquier tipo de disidencia doctrinal, social o científica podía ser mirada con sospecha. Esto se constata en el campo de la medicina, la cual era la única disciplina que había alcanzado un amplio desarrollo institucional y por ende se encontraba vigilada de forma celosa por sus autoridades y practicantes, por ello su apertura a las novedades era nula. En los tres siglos virreinales la medicina mostró una gran resistencia a las novedades; su enseñanza y práctica continuaron amarradas a los preceptos de los autores clásicos y su evolución fue bastante pobre. La obra de Diego Osorio y Peralta utiliza las mismas fuentes que se pueden encontrar en el primer libro de cirugía, la Suma y recopilación de cirngía de Alonso López de Hinojosos, publicado un siglo antes. Todavía en 1727, el Cursus medicus mexicanus del doctor Marcos José Salgado (1671-1740) presentaba un enfoque fisiológico fundamentado en la doctrina galénica y sus teorías ya eran en absoluto arcaicas para esa época (Trabulse, 1994: 43). Lo anterior, como se ha explicado, se debió a la política que emprendió la corona española a partir de la segunda mitad del siglo XVI y que tuvo una importante resonancia en la Nueva España.

La medicina académica hispánica de la Época Moderna tuvo una mayor refracción a las novedades debido a su avanzado estado de consolidación institucional, que la sujetaba a un control más estricto por parte de las autoridades reales. Por un lado estaban la Real Universidad y el Protomedicato, que se convirtieron en baluartes para controlar el conocimiento clásico de la medicina en el que las novedades eran rechazadas si concordaban con cualquier teoría, práctica o autor heterodoxo. Por el otro, se encontraba el Tribunal de la Inquisición, que entre sus múltiples funciones se encargaba de supervisar el contenido de las obras científicas. A este respecto, José Pardo Tomás ha señalado que una tercera parte de las obras con contenido científico que aparecieron en los índices de libros prohibidos durante los siglos XVI y XVII fueron libros de temas médicos (Pardo, 1991: 193). Aunque en muchas ocasiones los mecanismos inquisitoriales de censura presentaban fallas, su eficacia fue indefectible, lo que impidió el acceso a obras médicas provenientes de otras latitudes de Europa. De esta forma, la medicina hispánica, ejercida por un cuerpo de facultativos comprometidos con el escolasticismo tardío del siglo XVII, guareció su saber de las influencias intelectuales externas, haciendo que éste se mantuviera fiel a la tradición. Mientras tanto, en Europa, el conocimiento médico seguía otro derrotero que llevaría a nuevos descubrimientos anatómicos y a un más acelerado rompimiento con el paradigma galenista. 
GERARDO MARTÍNEZ HERNÁNDEZ

LA PRÁCTICA Y ENSEÑANZA DE LA ANATOMÍA EN LA REAL UNIVERSIDAD DE MÉXICO, SIGLOS XVI Y XVII

\section{CONCLUSIONES}

El estancamiento intelectual en España durante la Época Moderna ha sido un tema abordado en múltiples ocasiones, en cambio ese mismo fenómeno trasladado a los virreinatos americanos casi no ha recibido atención. La historia de la medicina novohispana ha carecido de un estudio comparativo que la pueda situar dentro del amplio contexto del mundo moderno. No hay duda de que el ambiente cultural de las colonias españolas se encontraba regido por los dictados de la metrópoli. Aquí es donde resulta interesante retomar lo apuntado por Elías Trabulse, quien asegura que la apertura hacia la ciencia moderna en la Nueva España sucedió en el segundo tercio del siglo XVII y fue un movimiento renovador que se dio a la par de la continuación de la ciencia verbalista y deductiva del neoescolasticismo. Sin embargo, bien lo señala el autor, esto se dio sólo en algunos campos de las ciencias, tales como la astronomía y las matemáticas (Trabulse, 1994: 41), que llegaban a escapar del total entendimiento de las autoridades inquisitoriales, en cambio, la medicina institucionalizada continuó por el camino del dogma.

A pesar del aparente pasmo intelectual de la medicina hispánica, hay situaciones que deben estudiarse con cautela. Como se ha visto, el conocimiento anatómico humano tuvo una importante transformación a mediados del siglo XVI. A partir de la imposición de la observación como fuente principal del conocimiento, el paradigma galénico comenzó a ser cuestionado y así se inició su lento declive. Sin embargo, el movimiento anatómico renovador, que tuvo como principal figura a Andrés Vesalio, no era sino la continuación de un desarrollo científico que se venía dando en algunos centros de enseñanza médica desde la Edad Media y que más tarde tuvo distintas vertientes en otras partes de Europa. ${ }^{25}$

25. Por ejemplo, en Italia se puede rastrear el desarrollo del conocimiento de la anatomía y fisiología a través de una sucesión de profesores de anatomía en la Universidad de Padua. Después de que Vesalio abandonó la cátedra anatómica en esta universidad, un discípulo suyo, llamado Gabriel Fallopio (1523-1562), la ocupó. Siguiendo el estilo vesaliano, Fallopio descubrió las «trompas de Fallopio» y continuó con la formación de excelentes anatomistas. Uno de sus alumnos, Girolamo Fabrizzio, o Hieronymus Fabricius ab Acquapendente (1537-1619) lo sustituyó en la docencia. Fabricius realizó una detallada descripción de las válvulas venosas, aunque no aportó ninguna idea original sobre la función de éstas, pues su explicación se encontraba dentro del esquema fisiológico del galenismo, el cual le daba una preponderancia al hígado sobre los demás órganos. Fabricius se retiró de la cátedra en 1613 por problemas de salud y finalmente murió en 1619. William Harvey (1578-1657) fue discípulo de Fabricius y heredero de una larga y rica tradición anatómica, que le permitió arribar al descubrimiento de la circulación de la sangre, teoría que expuso en su tratado Motu cordis et sanguinis in animalibus (1628). Antes de la aparición de esta obra se creía que la sangre se fabricaba en el hígado y era transportada a todo el cuerpo través de las venas, llevando alimento a los tejidos y se consumía totalmente en este proceso, de tal manera que se pensaba que se producía sangre nueva constantemente.

Ediciones Universidad de Salamanca / అ@@ Stud. his., H. ${ }^{a}$ mod., 42, n. 1 (2020), pp. 287-309 
En el caso particular de España, después de la incorporación de dos de los discípulos directos de Vesalio a las universidades hispánicas, Pedro Jimeno y Luis Collado, el movimiento anatómico renacentista en España no despegó. Esto se debió a distintos factores. Por un lado se puede mencionar la política contrarreformista iniciada por Felipe II para salvaguardar la rectitud religiosa. Y por otro, que fueron las circunstancias políticas y sociales las que llevaron a las autoridades e instituciones a ralentizar el desarrollo científico español.

En la Nueva España, a pesar de que se ejercía la disección anatómica desde muy tempranas fechas y de que se contaba con una mayor libertad en relación con las medidas inquisitoriales impuestas en la metrópoli, fue el mismo gremio médico, bajo la acción coercitiva de las autoridades, el que no se dio la oportunidad de continuar con un conocimiento anatómico innovador. Aunque llegaron médicos con una buena formación humanista, la pronta imposición de los dictados tridentinos los obligó a someterse a la ortodoxia. Además, la realidad americana implicaba condiciones poco favorables para el ejercicio intelectual. En primer lugar, los médicos novohispanos tuvieron que empezar a conformar un cuerpo colegiado que vigilara el ejercicio de la medicina y otras prácticas sanitarias en el virreinato y luego crear una facultad que brindara la docencia a las nuevas generaciones de médicos criollos. Ante el escaso desarrollo institucional, los pocos médicos que radicaban en la ciudad de México se dieron a la tarea de construir una base sólida para su profesión, dejando de lado el perfeccionamiento de nuevos conocimientos y tuvieron que atenerse a lo estipulado por los autores clásicos.

Aunque la medicina novohispana seguía las pautas de lo acontecido en la metrópoli, su situación geográfica le daba características e intereses propios. Los médicos novohispanos se mantuvieron alejados de las discusiones intelectuales de la medicina del Renacimiento. Sólo la obra Opera medicinalia (1570) de Francisco Bravo (c. 1530-1594), primer impreso médico del Nuevo Mundo (Martínez, 2011: 69-87), trató de entrar en los debates europeos, sin embargo, este libro no tuvo ninguna resonancia en el Viejo Continente. En cambio, las posteriores publicaciones médicas en la Nueva España, sobre todo los tratados de Alonso López de Hinojosos y de Agustín Farfán, definieron el modelo de medicina novohispana del siglo XVI más acorde con su realidad. En las páginas de estos libros se halla probablemente el conocimiento médico que era más común entre los galenistas y profesionistas sanitarios novohispanos y que se caracterizaba por un evidente mestizaje cultural, en el que el uso de las plantas medicinales americanas se hacía desde la óptica y apropiación de la teoría galénica. Esta medicina de carácter pragmático también contribuyó a la práctica de la disección anatómica durante las devastadoras epidemias del siglo XVI, en las que se realizaron disecciones anatómicas para intentar dar una explicación a la morbilidad y mortandad indígena. Pero aquellas prácticas anatómicas fueron un acontecimiento circunstancial. La enseñanza de la anatomía

Ediciones Universidad de Salamanca / 요 Stud. his., H. ${ }^{a}$ mod., 42, n. 1 (2020), pp. 287-309 
GERARDO MARTÍNEZ HERNÁNDEZ

LA PRÁCTICA Y ENSEÑANZA DE LA ANATOMÍA EN LA REAL UNIVERSIDAD DE MÉXICO, SIGLOS XVI Y XVII

fue establecida en el siglo XVII en la Real Universidad de México como un resabio de aquel intento de modernización de la teoría médica de mediados del XVI, pero ya bajo una perspectiva bastante conservadora. Para ese entonces la Nueva España había desarrollado ya una personalidad barroca propia, la cual se ve reflejada en la vida y obra de Diego Osorio y Peralta, autor del primer tratado de anatomía con fines docentes en la Real Universidad de México.

\section{BIBLIOGRAFÍA}

Barcia Goyanes, J. (1994), El mito de Vesalio, Valencia: Real Academia de Medicina de la Comunidad Valenciana/Universitat de Valencia.

Barón Fernández, J. (1970). Andrés Vesalio, su vida y su obra. Madrid: Instituto Arnaldo de Vilanova, CSIC.

Carlino A. (1999). Books of the body: anatomical ritual and Renaissance learning. Chicago: The University of Chicago.

Carreras Panchón. A (2005). Los saberes clásicos en su relación con la medicina europea. En L. E. Rodríguez-San Pedro Bezares y J. L. Polo Rodríguez (eds.). Saberes y disciplinas en las universidades hispánicas. Miscelánea Alfonso IX, 2004. Salamanca: Universidad de Salamanca, 97-109.

Ceballos Gómez, D. (1994). Hechicería, brujería e Inquisición en el Nuevo Reino de Granada. Un duelo de imaginarios. Medellín: Editorial Universidad Nacional.

Cunningham, A. (1997). The anatomical Renaissance: the resurrection of the anatomical projects of the ancients. Aldershot: Scholar Press.

Dávila Padilla, A. (1625). Historia de la fundación y discurso de la provincia de Santiago de México de la orden de los predicadores. Por la vida de sus varones insignes y casos notables de la Nueva España. Bruselas: en casa de Iván Meerbeque.

Estatutos y constituciones reales de la Imperial y Regia Universidad de México (1668). México: por la viuda de Bernardo de Calderón.

Farfán, A. (1592). Tratado breve de medicina, y de todas las enfermedades. México: en casa de Pedro Ocharte.

Fernández de Recas, G. (1960). Real y Pontificia Universidad de México. Medicina. Nómina de bachilleres, licenciados y doctores, 1607-1780. Guía de méritos y servicios, 1763-1828. Documentos del Archivo General de la Nación. México: UNAM.

Fernández del Castillo, F. (1953). La Facultad de Medicina según el Archivo de la Realy Pontificia Universidad de México. México: Imprenta Universitaria.

Galeno (2010). Del uso de las Partes. Madrid: Gredos.

González González, E. (1991). Proyecto de estatutos ordenados por el virrey Cerralvo. México: CESU-UNAM.

Grendler, P. (2002). The universities of the Italian Renaissance. Baltimore: Johns Hopkins University.

Hernández, F. (1959). Historia natural de la Nueva España. Vol. 1. México: Universidad Nacional Autónoma de México.

Ediciones Universidad de Salamanca / 요 Stud. his., H. ${ }^{a}$ mod., 42, n. 1 (2020), pp. 287-309 
GERARDO MARTÍNEZ HERNÁNDEZ

LA PRÁCTICA Y ENSEÑANZA DE LA ANATOMÍA EN LA REAL UNIVERSIDAD DE MÉXICO, SIGLOS XVI Y XVII

Jiménez Rueda J. (1951). Las Constituciones de la Antigua Universidad. México: Facultad de Filosofía y Letras.

Le Breton, D. (2002). La sociología del cuerpo. Buenos Aires: Ediciones Nueva Visión.

López de Hinojosos, A. (1977). Suma y recopilación de cirugía con un arte para sangrar muy útil y provechosa. México: Academia Nacional de Medicina.

López Piñero, J. M. (1976). La disección anatómica y la reforma vesaliana en la España del siglo XVI. En J. M. López Piñero, Medicina Moderna y sociedad española. Siglos $X V I-X I X$. Valencia: Cátedra e Instituto de Historia de la Medicina. Cuadernos Valencianos de Historia de Medicina y de la Ciencia, 61-130.

López Piñero, J. M. (1979). Ciencia y técnica en la sociedad española de los siglos XVI y XVII. Barcelona: Labor Universitaria.

Mandressi, R. (2012). La mirada del anatomista. Disecciones e invención del cuerpo en Occidente. México: Universidad Iberoamericana.

Martínez Hernández, G. (2008). La anatomía y la cirugía renacentistas en la Nueva España a través de la epidemia de cocoliztli y las figuras de Francisco Hernández, Alonso López de Hinojosos y Juan de la Fuente, 1576-1580. México: tesis de maestría, Facultad de Filosofía y Letras, UNAM.

Martínez Hernández, G. (2009). La repercusión de las reformas palafoxianas en la formación de los bachilleres médicos de la Real Universidad de México. En E. González, M. Hidalgo y A. Álvarez (coords.), Del aula a la ciudad. Estudios sobre la universidady la sociedad en el México virreinal. México: UNAM, pp. 87-106.

Martínez Hernández, G. (2011). El primer impreso médico del Nuevo Mundo: la Opera medicinalia del doctor Francisco Bravo, 1570. Intus Legere Historia, 5 (2), 69-87.

Martínez Hernández, G. (2014). Limpieza de sangre del doctor Juan de la Fuente, primer catedrático de medicina de la Real Universidad de México (1572). Estudios de Historia Novohispana, 50, 175-211.

Méndez, C. (1996). Libro del ejercicio corporal y de sus provechos. León: Ediciones Lancia.

Osorio y Peralta, D. (1685). Principia medicinae e epitome, et totius humani corporis fabrica seu ex microcosmi armonía divinum, germen. México: apud heredes viduae Bernardi Calderon.

Pardo Tomás, J. (1991). Ciencia y censura. La Inquisición española y los libros científicos en los siglos XVI y XVII. Madrid: CSIC.

Pardo Tomás, J. (2012). Opening Bodies in a New World: Anatomical Practices in Sixteenth-Century New Spain. En G. Olmi y C. Pancino (eds.), Anatome. Scomposizione, raffigurazione del corpo fra Medioevo e Etá Moderna. Bologna: Bononia University Press, 185-200.

Rodríguez-Sala, M. L. (1996). Diego Osorio de Peralta, hombre del barroco, autor del primer libro novohispano de anatomía como texto docente. Estudios de Historia Novohispana, 16 (16), 57-81.

Rodríguez-Sala, M. L. (1998). Los libros de medicina y de cirugía impresos en la Nueva España y sus autores durante los dos primeros siglos de cultura colonial (1570-1692). Segunda parte. Gaceta Médica de México, 134 (6), 723-725.

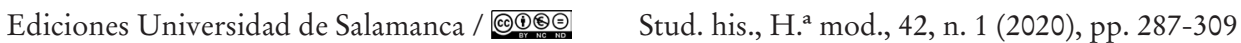


Rodríguez-San Pedro Bezares, L. E. (2010). La Universidad de Salamanca en la Monarquía hispánica: la fascinación de un nombre. En M. Soto, A. Velasco (coords.), Imperio, nación, Estado y diversidad cultural en Iberoamérica. Del orden colonial a las independencias. México: UNAM, 25-46.

Santander Rodríguez, T. (1965). La creación de la cátedra de cirugía en la Universidad de Salamanca. Cuadernos de Historia de la Medicina IV. Salamanca: Universidad de Salamanca.

Serrano Larráyoz, F. (2019). Graduados en Medicina por la Universidad de Irache (16131769). Arre-Pamplona: Pamiela (colección Universitas).

Skaarup, B. (2015). Anatomy and Anatomists in Early Modern Spain. Farnham: Ashgate.

Somolinos D’Ardois, G. (1980). Capitulos de historia médica mexicana. Relación alfabética de los profesionistas médicos, o en conexión con la medicina, que practicaron en territorio mexicano (1521-1618)(III). México: Sociedad Mexicana de Historia de la Medicina.

Trabulse, E. (1994). Los orígenes de la ciencia moderna en México (1630-1680). México: FCE.

Ediciones Universidad de Salamanca / 요 Stud. his., H. ${ }^{a}$ mod., 42, n. 1 (2020), pp. 287-309 